\title{
Reproducing kernel hilbert space based single infrared image super resolution
}

\author{
Liangliang Chen ${ }^{\mathrm{a}, 1}$, Liangjian Deng ${ }^{\mathrm{b}}$, Wei Shen ${ }^{\mathrm{c}}$, Ning Xi ${ }^{\mathrm{a}}$, Zhanxin $\mathrm{Zhou}^{\mathrm{a}}$, \\ Bo Song ${ }^{\mathrm{a}}$, Yongliang Yang ${ }^{\mathrm{a}}$, Yu Cheng ${ }^{\mathrm{a}}$, Lixin Dong ${ }^{\mathrm{a}}$ \\ ${ }^{a}$ Department of Electrical and Computer Engineering, Michigan State University, East \\ Lansing, MI 48824, USA \\ ${ }^{b}$ School of Mathematical Sciences, University of Electronic Science and Technology of \\ China, Chengdu, Sichuan, 611731, P.R. China \\ ${ }^{c}$ Department of Plant Biology, Michigan State University, East Lansing, MI 48824, USA
}

\begin{abstract}
The spatial resolution of Infrared (IR) images is limited by lens optical diffraction, sensor array pitch size and pixel dimension. In this work, a robust model is proposed to reconstruct high resolution infrared image via a single low resolution sampling, where the image features are discussed and classified as reflective, cooled emissive and uncooled emissive based on infrared irradiation source. A spline based reproducing kernel hilbert space and approximative heaviside function are deployed to model smooth part and edge component of image respectively. By adjusting the parameters of heaviside function, the proposed model can enhance distinct part of images. The experimental results show that the model is applicable on both reflective and emissive low resolution infrared images to improve thermal contrast. The overall outcome produces a high resolution IR image, which makes IR camera better measurement accuracy and observes more details at long distance.
\end{abstract}

Keywords: Infrared, Super resolution, Hilbert space, Reflective Infrared, Emissive Infrared

\footnotetext{
* Corresponding author

Email address: chenlia5@msu.edu (Liangliang Chen)
} 


\section{Introduction}

Infrared technology plays an important role in exploring beyond eye invisible imaging, which is connected with military needs [1, civilian application [2], scientific research 3 and medical treatment as traumatic knee injuries 4, can-

5 cer diagnostics [5]. As a result, it is estimated that the global infrared imaging market will reach to $\$ 8,450$ million by 2020 [ $]$ and the top players in the market are competing to penetrate the market by providing low cost, high resolution compact systems. The most critical component in infrared imaging system is sensor which determines the overall system performance. The sensing criteria can be divided as thermal and photon infrared sensing [7. The former sensitive element's temperature varies because of the IR absorption, which generates an electrical signal change. The latter photon infrared detectors absorb infrared radiation and induce electrons into a higher energy level which contributes to electric signal. The photon quantum detectors are very fast response $(n s-\mu s)$ compared to thermal IR detector $(m s)$ [ $]$. Although the cost, speed, sensitivity and temperature range are key features for infrared imaging system, the spatial resolution determines the fine detail and clarity, the temperature measurement accuracy, imaging distance and object size [9. There are at least three factors hindering high resolution IR Focal Plane Array (FPA): optical diffraction, readout circuit (ROIC) mismatch and detector non-uniformity [10. In the visible spectrum, silicon can be both used for sensing material and readout electronics. However, infrared FPA is fabricated by $\mathrm{Hg}_{1-x} \mathrm{Cd}_{x} \mathrm{Te}, \mathrm{Pb}_{1-x} \mathrm{Sn}_{x} \mathrm{Te}, \mathrm{InSb}$, AlGaAs etc [1]. In order for integration, the FPA and ROIC are separate in hybrid array construction, where two arrays are carefully aligned and pressed together using indium as electrical connection [12. For the flip-chip hybrid technology, the chip size is limited on order of $20 \mathrm{~mm}^{2}$ due to thermal mismatch between FPA and ROIC [9]. Although there is non-uniformity correction based on-chip processing [13] to reduce the static spatial pattern noise and alternative epitaxy technology with sapphire to overcome thermal mismatch problem, the hardware cost is still unacceptable high. 
From signal processing perspective, obtaining High Resolution (HR) image from observed Low Resolution (LR) image/images is an interpolation problem which expands low frequency sampling into high bandwidth [14. Table 1 lists the super resolution method in visible spectrum. The most straightforward is nonuniform interpolation [15, which is performed successively by motion estimation, interpolation and restoration. In [16], the aliasing between LR and HR signals fourier transformation is made to reconstruct super resolution image in frequency domain. The model based approaches including regularized reconstruction, deterministic [17, and stochastic [18] method are also reported, though all are high computational cost. Single LR image based super resolution (SR) algorithm is more competitive compared to multiple LR images method due to easy system integration. The nearest neighbor [19] and bicubic interpolation [20] are widely used in imaging tools. The machine learning based super resolution needs large training set to output a better result. Meanwhile, the 45 novel approaches focus on compressive sensing [21] and sparse representations 22] 223] to improve image spatial resolution.

The infrared image super resolution is more challenging and essential due to high cost manufacturing process for large sensor array in MWIR and LWIR wavelength. The earlier IR super resolution methods were coming from visible 50 spectrum techniques [24] 25]. Besides, The sparse representation was also applied on infrared image super resolution which made the high resolution patches share the same representation as low resolution patches [23]. The non-sampled contourlet transform was proposed to improve infrared image quality based on low pass subband and bandpass subband images [26. The regularized technique was adopted on infrared images super resolution by $\ell_{1}$-norm and total variation norm [27. The human vision processing mechanism together with vision lateral inhibition was utilized to enhance the image contrast between object and background 28]. Moreover, the correlation of an infrared image and its corresponding visible image were suggested to improve resolution, in which the visible image generated edge maps [29. In overall, most approaches were directly borrowed from visible image processing which didn't consider the features 
Table 1: Visible spectrum image super-resolution.

\begin{tabular}{|c|c|c|}
\hline Method & $\begin{array}{l}\text { Obeserved } \\
\text { LR Images }\end{array}$ & Characteristics \\
\hline Nonuniform interpolation & Multiple & $\begin{array}{l}\text { Low computational load and } \\
\text { real time application possible }\end{array}$ \\
\hline Frequency domain & Multiple & $\begin{array}{l}\text { Theoretical simplicity, convenient } \\
\text { for parallel implementation }\end{array}$ \\
\hline Regularized reconstruction & Multiple & Inversion of ill-posed problem \\
\hline Deterministic approach & Multiple & $\begin{array}{l}\text { Minimize the amount of high } \\
\text { pass energy in the restore image }\end{array}$ \\
\hline Stochastic & Multiple & $\begin{array}{l}\text { Quadratic potential function, } \\
\text { solution become oversmoothed }\end{array}$ \\
\hline Nearest-neighbor & Single & Fast but jaggy effect \\
\hline Bicubic interpolation & Single & Blur effect \\
\hline Learning based & Single & $\begin{array}{l}\text { Training data set, computational } \\
\text { expensive }\end{array}$ \\
\hline
\end{tabular}


Table 2: IR irradiation in night scene.

\begin{tabular}{llll}
\hline Wavelength & Type & Category & IR Camera Application \\
\hline $0.72-1.4$ & Reflective & Near IR & Night vision goggles \\
$1.4-3$ & Reflective/Emissive & SWIR & Night vision camcorder \\
$3-8$ & Emissive & MWIR & MWIR Camera \\
$8-14$ & Emissive & LWIR & LWIR Camera \\
\hline
\end{tabular}

of infrared image.

In this paper, a single image based super resolution method is proposed to reconstruct high resolution IR image, which is robust on near IR, SWIR, MWIR and LWIR images. The distinct features of IR image are classified as reflective, cooled emissive and uncooled emissive by sensing mechanism. Section 2 briefly describes the characteristic of IR images from reflection and emission. Section 3 introduces the low resolution observation model and how to build mathematical model using Reproducing Kernel Hilbert Space (RKHS) and heaviside function. Section 4 discusses the algorithm in details. The experimental results and conclusions are depicted in section 5 and section 6 .

\section{Characterization of IR image}

The IR images measure infrared irradiation and its distributions. There are two IR sources for imaging, one is internal emissivity due to Planck's law 75 [7] and another is external reflection similar as visible light. In other words, they are working in passive mode, which detects the thermal radiation given off by a warm object without the need for illumination, or active mode, which uses an invisible infrared source to illuminate the scene. Table 2 compares the wavelength, working type and its applications. The reflective and emissive are corresponding to active mode and passive mode respectively.

\subsection{Reflective IR image}

In natural, there are a lot of IR sources, as sunlight and airglow at night, man-made LED and laser IR illuminators, which irradiate near IR about $750 \mathrm{~nm}$ 
to $1 \mu \mathrm{m}$ in wavelength. The active/reflective infrared imaging system behaves exactly similar as visible SLR camera, which expresses the shape of object and its infrared reflectance. However, there is a considerable difference between visible and near IR images. The near IR reflectivity is quite different from visible for most of materials. As shown in Figure 1, the vegetation and dry bare soil have higher reflectivity in near IR than visible while water has all absorption in near 90 IR band. In Overall, the near IR images have a higher contrast for outside imaging [30]. Figure 2 shows the difference between visible (right) and near IR (left) reflection image on same scene, in which the brightness and contrast of left (near IR) images are higher than right (visible) in visual. The sharpness and contrast are more important and dominant features on near IR images.

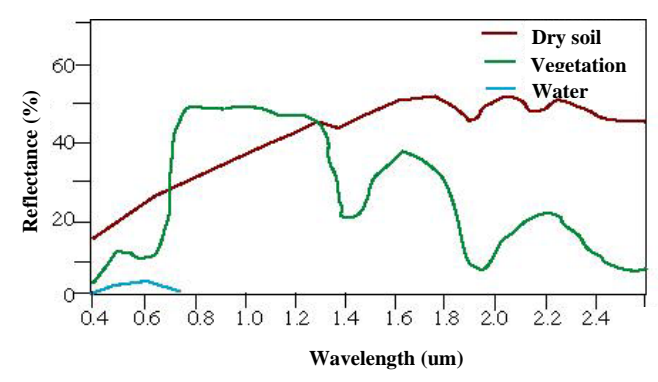

Figure 1: The reflectance on different materials

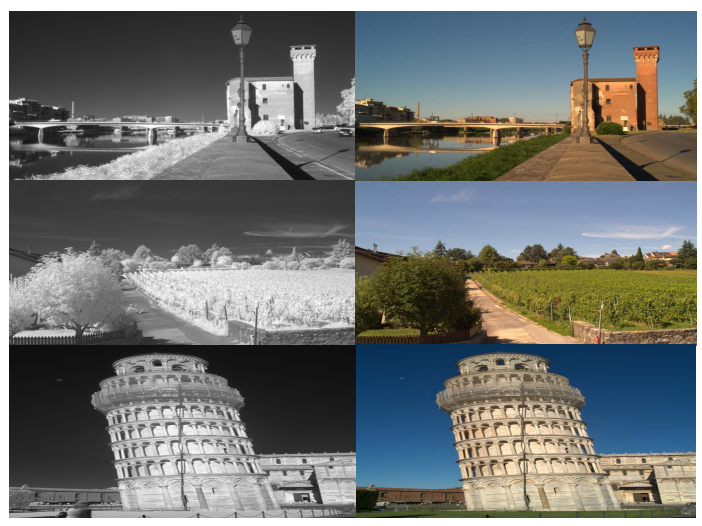

Figure 2: The comparison between near IR reflective image and visible image on same scene 
The reflective IR imager limits the application due to IR source needed. However, the image acquisition in passive/emissive mode, also referred to thermal infrared camera, consists of another huge number of applications. It measures the emissivity and temperature of objective target comprehensively. The

100 thermal infrared spectrum spreads from MWIR to LWIR, VLWR for nature scene. From photodetector perspective, there are narrowband cooled and broadband uncooled, depending on FPA sensing wavelength and materials. The narrowband cooled IR camera is photon based, as all intrinsic materials, III-V compounds (InGaAs, InAsSb, InGaSb), extrinsic materials, Si:As, Si:Sb hybrid arrays, and quantum well structures, GaAs/AlGaAs QWIPs [9]. In order to sense such small photon energy and reduce thermal noise, the photon IR detector needs cryogenics cooling [7. The sensors have very faster response speed, larger spatial resolution and higher thermal contrast to explore tiny object, especially with filtering technique [31. However, on the state of art commercial IR photon based cameras, they are bulky and expensive although they provide better image quality. Besides, the non-uniformity is more challenging due to manufacturing defects and darkcurrent [10]. Firstly, each sensor unit performs differently. Secondly, the sensing element and readout circuits are separate as a result of non-compatible process between silicon and other materials technology.

The broadband uncooled IR sensors absorb wide spectrum and function in the LWIR where most of energy is emitted by terrestrial targets. The sensor array senses the scene temperature change without cooling. They measure the temperature or its gradient, including micromachined silicon bolometers, pyroelectric detectors, barium strontium titanate (BST) and lead titanate $\left(\mathrm{PbTiO}_{3}\right)$ and ferro-electric sensors [8]. The temperate measurement is such slow because of a heat sink connected. Although spinning wheel chopper was introduced to modulate the signal for ferroelectric detectors, it would lead to extra halo effects on images [10. The thermal contrast on broadband uncooled thermal images are quite lower than narrowband cooled approach. Some materials such as metal, have very high reflectivity for thermal radiation so that the surround- 
ing temperature can reflect on the desired target. The reflective temperature becomes important in thermal temperature imager.

\section{Theory of proposed approach}

The image contrast and spatial resolution are two figure of merits to characterize infrared camera [7]. The contrast is determined by resolution and sensitivity measured by Noise Equivalent Temperature Difference (NETD). The resolution is dependent on focal plane array size and closely related to undersampling and spatial aliasing. The high resolution measurements not only see and understand more accurately but also resolve small details in long distance observation.

\subsection{Low resolution sampling}

The originates of low resolution sampling for active (reflective) and passive (emissive) IR camera are distinct in essential. As discussed in Table 2, the reflected wavelength is always in near IR or short wave IR, in which the detector process is compatible with silicon manufacturing. In active IR camera, the most directly way to enhance resolution is to increase the chip size (together with lens changes) or reduce the pixel size to increase the number of pixels in unit area. The disadvantage of former method is to increase capacitance which makes the readout circuit in low charge transfer rate. It also requires a high precision optics 32 . For the latter solution, reducing the pixel size will also decrease the amount of light coming to each sensor so that the shot noise will degrade the image quality. As shown in Eq.(1), the reflected illumination is collected by sensor array in a limited space-time volume. The induced electrons accumulated on each pixel is reverse proportional to the square ratio of focal length to aperture of lens $(F)$, but proportional to exposure time $(t)$, incident illumination $\left(I_{s r c}\right)$, scene reflectivity $(R)$, quantum efficiency $(q)$ and pixel size $\left(\Delta^{2}\right)$ 33.

$$
\mathbf{J}=10^{15}(F)^{-2} t I_{s r c} R q \Delta^{2}
$$


The passive IR images have technical obstacles to get a ultra high spatial resolution in hardware perspective. Both cooled and non-cooled cameras are limited by non-uniformity fabrication and aliasing sampling. In IR camera optics, the spatial resolution is determined by pixel pitch size (D), which is limited by diffraction of optics as Eq. 22 , where $f$ is focal ratio, $f$-stop, or relative aperture, $\lambda$ is selected wavelength. The industry standard pitch is $17 \mu m$. For example, the CMT-640L camera use 8-9.4 $\mu \mathrm{m}$ band with the pixel of $16 \times 16 \mu \mathrm{m}$. The longer wavelength Sofradir SSC-320VL employs $30 \times 30 \mu m$ MCT detector. The pixel pitch of infrared sensors is far larger than visible camera. Although many camera manufacturers employ IR detectors that use smaller pixel sizes than what is theoretically needed, it will oversample the signal but not result in increasing resolution. Because of the diffraction limit, the detectors used in SWIR, MWIR, and LWIR have respectively larger pixel sizes and lower spatial resolution capabilities.

$$
\mathbf{D}=(2.44) \lambda \times f
$$

\subsection{Observation modeling}

The digital IR image acquiring is highly hardware dependent. The output image comes with various kinds of degradations from motion blur, aliasing and noisy version of true scene [14]. As shown in Eq.(3), $\mathbf{X}=\left[x_{1}, x_{2}, \ldots, x_{N}\right]$ denotes the desired high resolution image above nyquist sampling frequency. $\mathbf{Y}$ is the single LR image observed. $B$ is the blur effect model and $D$ is the down sampling operator. $n$ represents the noise model. The LR image is denoted as $\mathbf{Y}(s, t),\left(s=1,2, \ldots N_{1}, t=1,2, \ldots N_{2}\right), N_{1}, N_{2}$ are horizontal and vertical resolution of LR image. The parameters $L_{1}, L_{2}$ are down sampling factors for each direction respectively and $N=L_{1} N_{1} L_{2} N_{2}$. In mathematically, blur matrix $B$ is $L_{1} N_{1} L_{2} N_{2} \times L_{1} N_{1} L_{2} N_{2}$, while subsampling matrix $D$ is $\left(N_{1} N_{2}\right)^{2} \times L_{1} N_{1} L_{2} N_{2}$ size. The SR method is to estimate $\mathbf{X}$ in Eq.(3) by optimization with con- 
straints.

$$
\begin{array}{r}
\mathbf{Y}=D B \mathbf{X}+n, \\
\mathbf{X}=\left[x_{1}, x_{2}, \ldots, x_{N}\right]
\end{array}
$$

\subsection{Proposed Model}

The image intensity $f(x, y)$ is divided by smooth component and edges, as $f(x, y)=h(x, y)+g(x, y)$. The smooth part, $h(x, y)$ is modeled by functions in a Reproducing Kernel Hilbert Space (RKHS) and every evaluation function is bounded. Edge is described as step function in $g(x, y)$.

To simplify the analysis, 1-D signal is introduced in the model. For an arbitrary real value $h(x), x \in[0,1]$, its Taylor series can be expanded at $x=0$ as Eq.(4). $h_{0}(x)$ and $h_{1}(x)$ is corresponding to first and second part of $h(x)$, also referred to lagrange remainder. The span of Eq.(5) will form a RKHS [34, $\mathbb{H}_{0}=\operatorname{span}\left\{\phi_{1}, \phi_{2}, \phi_{3}, \ldots, \phi_{m}\right\}$ and $h_{0}(x)=\sum_{n=1}^{m} d_{n} \phi_{n}(x), d_{n}(n=1,2, \ldots, m)$ is coefficient.

$$
\begin{gathered}
h(x)=\sum_{n=0}^{m-1} \frac{x^{n}}{n !} h^{(n)}(0)+\int_{0}^{1} \frac{(x-t)^{m-1}}{(m-1) !} h^{(m)}(t) d t \\
=h_{0}(x)+h_{1}(x) \\
\phi_{n}(x)=\frac{x^{n-1}}{(n-1) !} \\
n=1,2,3, \ldots, m
\end{gathered}
$$

The remainder $h_{1}(x)$ can also be expanded on a RKHS 34. Let $G_{m}(x, u)=$ $\frac{(x-u)^{m-1}}{(m-1) !}$ and the boundary conditions $h_{1}^{n}(0)=0,(n=0,1, \ldots, m-1) . \mathbb{H}_{1}$ is a Hilbert space on $[0,1]$ with reproducing kernel $K(s, t)=\int_{0}^{1} G_{m}(t, u) G_{m}(s, u) d u$ [35. The function $h_{1}$ can be expanded on the basis of $\mathbb{H}_{1}, h_{1}(x)=\sum_{i=1}^{n} c_{i} K\left(s_{i}, x\right)$.

The generalized taylor series expansion is described as Eq. (7). In matrix format, $\vec{h}=T d+\Sigma c$, where $T$ is $n \times m$ matrix and $\Sigma$ is a $n \times n$ matrix and 
$d=\left(d_{1}, d_{2}, \ldots, d_{m}\right)^{\prime}, c=\left(c_{1}, c_{2}, \ldots, c_{n}\right)^{\prime}$. The noise observation $\vec{g}=\vec{h}+\eta$ can be smoothing by minimizing Eq. (8).

$$
\begin{aligned}
h_{1}(x) & =\int_{0}^{1} \frac{(x-u)^{m-1}}{(m-1) !} h^{(m)}(u) d u \\
& =\int_{0}^{1} \frac{(x-u)^{m-1}}{(m-1) !} d h^{(m-1)}(u) \\
& =G_{m}(x, 1) h^{(m-1)}(1)-G_{m-1} h^{(m-2)}(1)+ \\
G_{m-2}(x, 1) h^{(m-3)}(1)+. .+G_{1}(x, 1) h^{(0)}(1) & \\
& h(x)=\sum_{v=1}^{m} d_{v} \phi_{v}(x)+\sum_{i=1}^{n} c_{i} \xi_{i}(x) \\
& \min _{c, d} \frac{1}{n}\|\vec{g}-T d-\Sigma c\|^{2}+\lambda c^{\prime} \Sigma c
\end{aligned}
$$

2D thin plate spline based RKHS is similar as 1D [35. The null space is spanned by polynomials, $\phi_{1}(x, y)=1, \phi_{2}(x, y)=x, \phi_{3}(x, y)=y, \phi_{4}(x, y)=$ $x y, \phi_{5}(x, y)=x^{2}, \phi_{6}(x, y)=y^{2}$. Eq. 9 shows the $2 \mathrm{D}$ unique solution. The matrix format is as Eq. (8), where $\mathrm{T}$ is $n \times M$ matrix, $\Sigma$ is a given $n \times n$ matrix. $d=\left(T^{\prime} W^{-1} T\right)^{-1} T^{\prime} W^{-1} \mathbf{g}, c=W^{-1}\left(I-T\left(T^{\prime} W^{-1} T\right)^{-1} T^{\prime} W^{-1}\right) g, W=\Sigma+n \lambda I$.

$$
h(x, y)=\sum_{v=1}^{m} d_{v} \phi_{v}(x, y)+\sum_{i=1}^{n} c_{i} \xi_{i}(x, y)
$$

As previous discussions, the RKHS based model makes the image smooth. However, the image contrast is edge dependent and functions significantly, especially for narrow band cooled passive IR camera. The heaviside step function includes a singular point at $x=0$ which describes edge on image signal perfectly, as shown in Eq.10. In the proposed model, an approximated heaviside function, Eq.11 is applied, where $\xi$ controls the sharpness of signal. Figure 3 compares the heaviside function and two approximated heaviside functions 
under $\xi=0.6$ and $\xi=0.04$, where $\xi$ controls the smoothness and the smaller $\xi$ has shaper edge.

$$
\begin{gathered}
\phi(x)= \begin{cases}0 & x<0 \\
1 & x \geq 0\end{cases} \\
\psi(x)=\frac{1}{2}+\frac{1}{\pi} \arctan \left(\frac{x}{\xi}\right)
\end{gathered}
$$

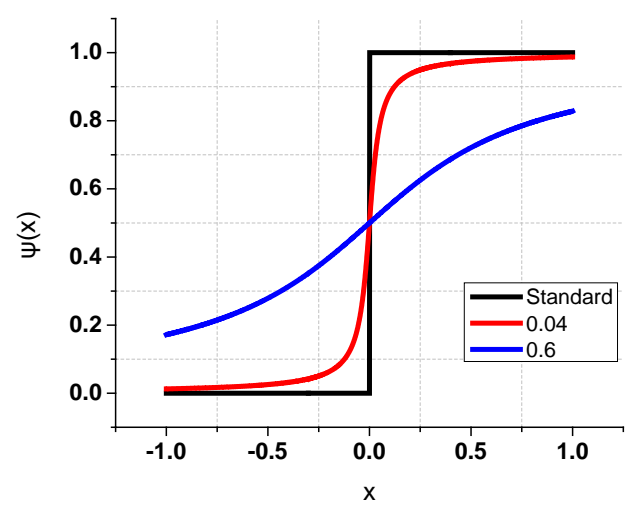

Figure 3: Heaviside function - black, approximated heaviside functions with 0.04 (red solid line) and 0.6 (blue line).

The 2D approximate heaviside function is easily converted by Eq. 111. Let $\mathbf{v}_{i}=(\cos \theta, \sin \theta), \psi\left(\mathbf{v}_{i} \cdot \mathbf{x}+c_{i}\right)\left(\mathbf{x} \in \mathbb{R}^{2}\right)$ represents an edge with orientation $\theta_{i}$ located at position $\mathbf{x}$ with offset $c_{i}$. As $\theta_{i}$ and $c_{i}$ vary, the image edge can be modeled using Eq. 12$\}$, where $\theta_{j} \in\{0, \pi / 12,2 \pi / 12,3 \pi / 12, \ldots, 23 \pi / 12\}$, $c_{j} \in\left\{0, \frac{1}{n-1}, \frac{2}{n-1}, \ldots, 1\right\}, \beta_{j}$ is coefficient. In this work, the number of orientations is 24 and the number $m$ at Eq. 12 equals to total pixels of LR divided by 24 . In matrix format, $g=\Psi \beta, \Psi \in \mathbb{R}^{n \times m}, \beta \in \mathbb{R}^{m}$. Meanwhile, $\xi$ controls the sharpness of edge. It is selected distinctly for reflective IR image, narrow band and broadband emissive IR images depending on the image contrast requirement. The total image intensity function is the summation of edges and 
smooths part. $\vec{f}=\vec{h}+\vec{g}=T d+\Sigma c+\Psi \beta$, where $\vec{g}$ is edge model, $\Psi$ is edge matrix and $\beta$ is edge coefficients.

$$
\begin{array}{r}
g(\mathbf{x})=\sum_{j=1}^{m} \beta_{j} \psi\left(\mathbf{v}_{j} \cdot \mathbf{x}+c_{j}\right) \\
\mathbf{v}_{j}=\left(\cos \theta_{j}, \sin \theta_{j}\right)
\end{array}
$$

\section{Iterative Reconstruction Algorithm}

Based on proposed model, the super resolution image restoration is a minimization problem, as Eq. 13, where desired $H=T^{h} d+\Sigma^{h} c+\Psi^{h} \beta$ is the target high resolution output. Since $\|\beta\|_{1}$ is not differentiable, the equivalent problem is described in Eq. (14) by substituting $\beta$ as $u$. The constraint equation $\ell_{1}$ problem can be easily converted to its augmented lagrangian function as Eq.(15), $\alpha, \rho, b \in \mathbb{R}$. In order to solve Eq. 15 , it is divided by two subproblems as $u$ problem and $(d, c, \beta)$ problem. The $u$-subproblem, Eq.116 has the solution of Eq. [17] [36.

$$
\begin{gathered}
\min \frac{1}{n}\left\|L-D B\left(T^{h} d+\Sigma^{h} c+\Psi^{h} \beta\right)\right\|^{2}+\lambda c^{\prime} \Sigma c+\alpha\|\beta\|_{1} \\
\min \frac{1}{n}\left\|L-\left(T^{l} d+\Sigma^{l} c+\Psi^{l} \beta\right)\right\|^{2}+\lambda c^{\prime} \Sigma^{l} c+\alpha\|u\|_{1} \\
\text { s.t., } u=\beta \\
\mathbb{L}(d, c, \beta, u)=\frac{1}{n}\left\|L-\left(T^{l} d+\Sigma^{l} c+\Psi^{l} \beta\right)\right\|^{2}+\lambda c^{\prime} \Sigma^{l} c+\alpha\|u\|_{1}+\frac{\rho}{2}\|u-\beta+b\|^{2} \\
\min \alpha\|u\|_{1}+\frac{\rho}{2}\|u-\beta+b\|^{2} \\
u_{i}=\max \left\{\left\|\beta_{i}-b_{i}\right\|, \frac{\alpha}{\rho}\right\} \frac{\beta_{i}-b_{i}}{\left\|\beta_{i}-b_{i}\right\|}
\end{gathered}
$$


Table 3: RKHS based single image super resolution algorithm.

Algorithm
for $\mathbf{i}=\mathbf{1}: \mathbf{n}$
a. Compute the $u^{(i)}$ by Eq. 17
b. Update the coefficients: $\left(d^{(i)}, c^{(i)}, \beta^{(i)}\right)=$
argmin $\frac{1}{n}\left\|L^{(k)}-\left(T^{l} d+\Sigma^{l} c+\Phi^{l} \beta\right)\right\|^{2}+\lambda c^{\prime} \Sigma^{l} c+\alpha\|\beta\|_{1}$
c. Calculate the high-resolution image:
$H^{(k)}=T^{h} d^{(k)}+\Sigma^{h} c^{(k)}+\Phi^{h} \beta^{(k)} \cdot$
d. Down sampling to $\tilde{L}=D B H^{(k)}$.
e. Residual update: $L^{(i+1)}=L^{(k)}-\tilde{L}$
end

The $(d, c, \beta)$-subproblem is more complicated than $u$ - subproblem. In this work, the Eq.18 subproblem can be employed by least square method [34] so that $d, c, \beta$ can be obtained. The main iteration is shown in Table 3.

$$
\min _{d, c, \beta} \frac{1}{n}\left\|L-\left(T^{l} d+\Sigma^{l} c+\Psi^{l} \beta\right)\right\|^{2}+\lambda c^{\prime} \Sigma^{l} c+\frac{\rho}{2}\|u-\beta+b\|^{2}
$$

\section{Experimental Results}

In this section, there are three groups of numerical experiments discussed: reflective/active IR images, narrow band cooled and broadband uncooled IR emissive/passive images. The ground truth images come from IR camera. In order for comparison and algorithm evaluation, a low resolution image is generated from downsampling of HR image at scale of 2. All experiments are run in MATLAB(R2010b) on a laptop of 8Gb RAM and Intel(R)Core(TM) i7-4510U CPU: $2.60 \mathrm{GHz}$.

The first numerical experiment is run on near IR images, which work similar as SLR camera but different wavelength. The reflective image manifests the object shape and material reflectance, especially on infrared spectrum. The image detail (high frequency components, the edges) and outline (low frequency, 
smooth variations) are equivalent, so that a medium edge part is chosen to model it.

As shown in Figure 4, there are 8 recovered images listed for comparison, including bicubic, bilinear, nearest, edge adaptive interpolation by local orientation of image contours (SPIE09) [37], sparse mixing estimators (TIP10) 38, sparse representation based image interpolation with nonlocal autoregressive modeling (TIP13) 39], statistical prediction sparse representations (TIP14) 40] and proposed method. Two groups near infrared images are discussed herein, plant tissue and building as Figure 4 and Figure 5 . Since it is difficult to distinguish the tiny difference in visual, the Root Mean Square Error (RMSE), Peak Signal to Noise Ratio (PSNR) and Structural similarity (SSIM) index are compared in Table 4. It is obviously that the proposed method has better performance (less RMSE, larger PSNR and SSIM). In both near IR images (Table 4 and Table 5), the results of bicubic and bilinear interpolation show significant blur effect. The nearest approach introduces much jaggy effect on the edge. Although the outcome by SPIE09 and TIP10 keep sharp edges well, they make image details of non-edge regions oversmoothing. In overall, the learning-based method (TIP14) obtains the best performance (smallest RMSE and largest PSNR and SSIM). The proposed method preserves image details of non-edge regions better and generate ranked second output in quantitatively.

\begin{tabular}{llll} 
Table 4: RMSE, PSNR and SSIM of near IR image - Plant tissu \\
\hline Enhanced method & RMSE & PSNR & SSIM \\
\hline Nearest & 7.6283 & 30.4822 & 0.9948 \\
Bicubic & 5.8892 & 32.7297 & 0.9954 \\
Bilinear & 6.9002 & 31.3535 & 0.9899 \\
SPIE09 & 8.8736 & 29.1688 & 0.9709 \\
TIP10 & 8.1671 & 29.8894 & 0.9794 \\
TIP13 & 5.6128 & 33.1472 & 0.9845 \\
Proposed Method & 4.8457 & 34.4237 & 0.9981 \\
TIP14 & 4.6653 & 34.7532 & 0.9986 \\
\hline
\end{tabular}




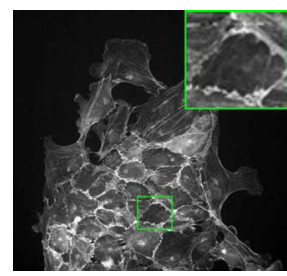

(a)

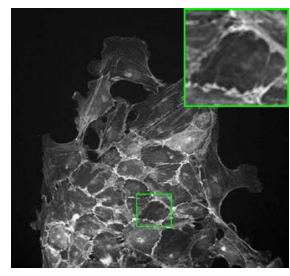

(e)

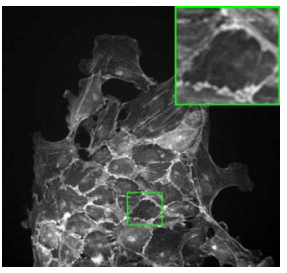

(b)

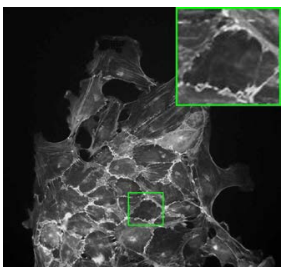

(f)

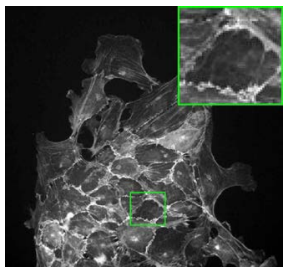

(c)

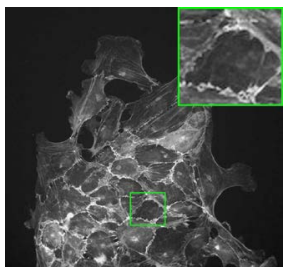

(g)

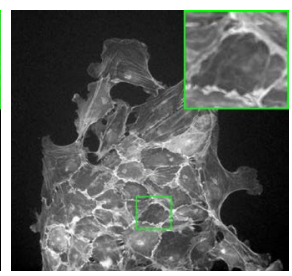

(d)

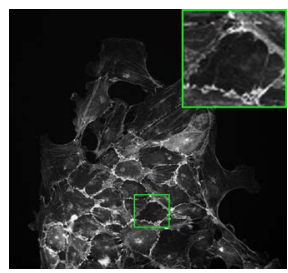

(h)

Figure 4: Plant tissue near IR image. (a) bicubic, (b) bilinear, (c) nearest neighbor, (d) SPIE09, (e) TIP10, (f) TIP13, (g) TIP14, (h) proposed method

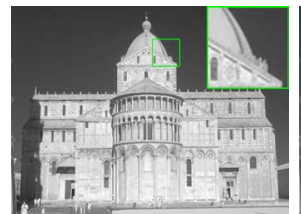

(a)

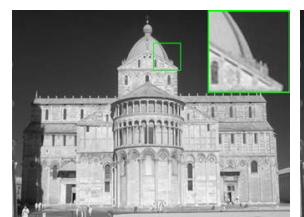

(e)

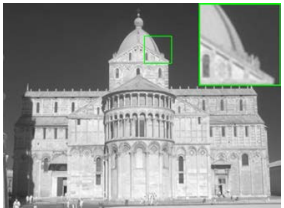

(b)

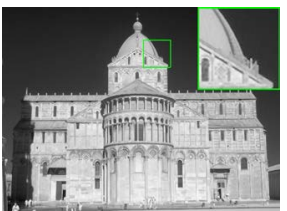

(f)

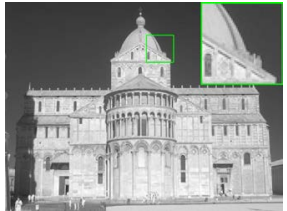

(c)

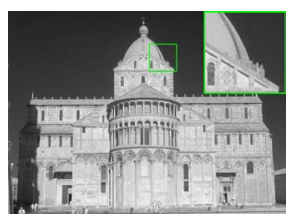

(g)

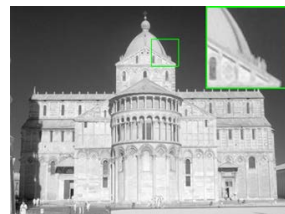

(d)

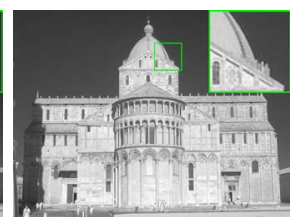

(h)

Figure 5: Building near IR image. (a) bicubic, (b) bilinear, (c) nearest neighbor, (d) SPIE09, (e) TIP10, (f) TIP13, (g) TIP14, (h) proposed method

The cooled emissive IR images have ultra high sensitivity and background filter so that they bring a high contrast with great sharpness. However, the smooth part dominates super resolution result since the edge can be sampled in low resolution image. The proposed model is modified with a smaller edge component compared to reflective IR image super resolution. Figure 6 and Figure 7 


\begin{tabular}{llll}
\multicolumn{4}{c}{ Table 5: RMSE, PSNR and SSIM of near IR image - Building. } \\
\hline Enhanced method & RMSE & PSNR & SSIM \\
\hline Nearest & 8.7152 & 29.3253 & 0.9939 \\
Bicubic & 6.9646 & 31.2729 & 0.9945 \\
Bilinear & 7.9934 & 30.0762 & 0.9880 \\
SPIE09 & 8.1188 & 29.9410 & 0.9767 \\
TIP10 & 9.1753 & 28.8784 & 0.9778 \\
TIP13 & 6.5503 & 31.8088 & 0.9900 \\
Proposed Method & 6.2632 & 32.1949 & 0.9963 \\
TIP14 & 5.4804 & 33.0546 & 0.9980 \\
\hline
\end{tabular}

show tow cooled emissive IR images and their super resolution comparison. As compared in Table 6 and Table 7, the nearest neighbor has worst reconstruction since it introduces errors on edge. The errors on SPIE09 and TIP10 come with oversmoothing on the edge. However, the proposed approach and TIP14 (learning based) produce better results with SSIM larger than 0.99. These prove that the oversmoothing algorithm will not improve cooled emissive IR image quality.

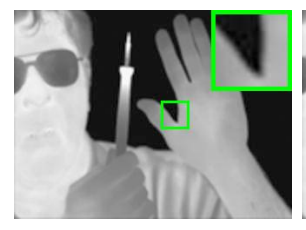

(a)

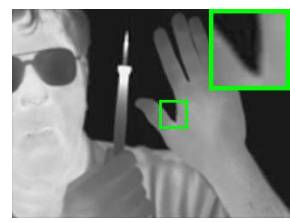

(e)

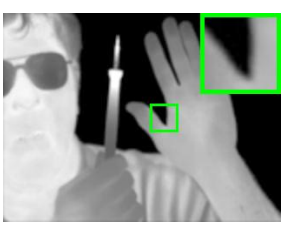

(b)

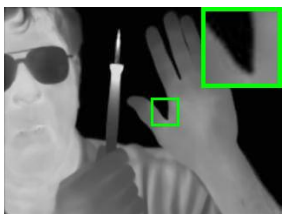

(f)

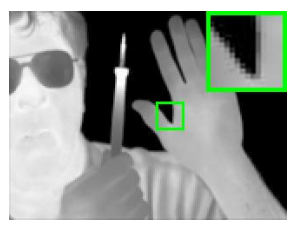

(c)

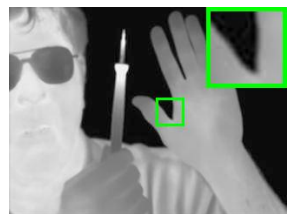

(g)

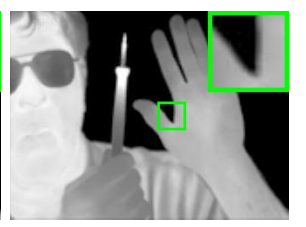

(d)

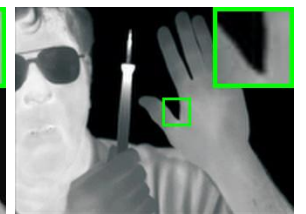

(h)

Figure 6: Human cooled emissive IR image. (a) bicubic, (b) bilinear, (c) nearest neighbor, (d) SPIE09, (e) TIP10, (f) TIP13, (g) TIP14, (h) proposed method

The uncooled IR images are not as good as cooled sensors because of low sen- 
Table 6: RMSE, PSNR and SSIM of cooled emissive IR image - human.

\begin{tabular}{llll}
\hline Enhanced method & RMSE & PSNR & SSIM \\
\hline Nearest & 6.0111 & 32.5518 & 0.9631 \\
Bicubic & 3.2502 & 37.8925 & 0.9877 \\
Bilinear & 3.9260 & 36.2518 & 0.9822 \\
SPIE09 & 5.9357 & 32.6613 & 0.9681 \\
TIP10 & 5.6335 & 33.1353 & 0.9688 \\
TIP13 & 3.6134 & 36.9725 & 0.9892 \\
Proposed Method & 2.9484 & 38.7391 & 0.9917 \\
TIP14 & 2.5093 & 40.1398 & 0.9947 \\
\hline
\end{tabular}

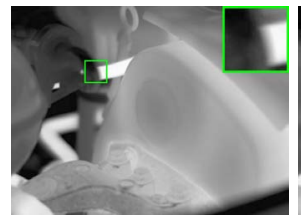

(a)

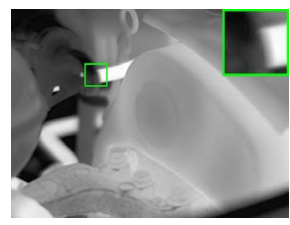

(e)

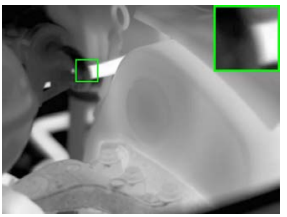

(b)

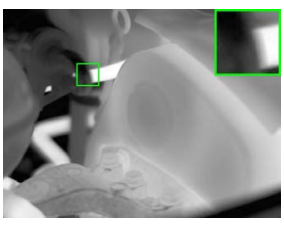

(f)

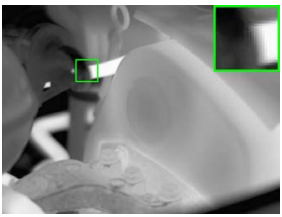

(c)

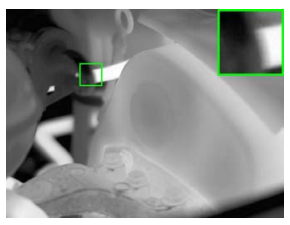

(g)

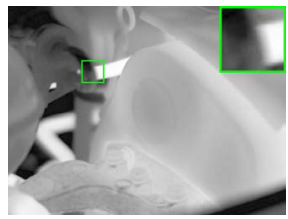

(d)

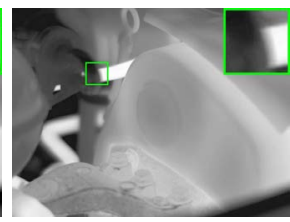

(h)

Figure 7: Car engine cooled emissive IR image. (a) bicubic, (b) bilinear, (c) nearest neighbor, (d) SPIE09, (e) TIP10, (f) TIP13, (g) TIP14, (h) proposed method

sor sensitivity or external noise as halo effect [10. The image thermal contrast is lower than cooled images so that the edge would be lost in low resolution sampling. In addition, there are background noise as shown in Figure 8 and Figure 9. Table 8 and Table 9 compare the performance of recovery, where the SSIM is ordered from worst to best value (closest to 1). Because of random background noise, TIP14 doesn't generate best output. It is very hard to find a generic algorithm on noisy thermal IR images. Compared to these two numerical analysis (Table 8 and Table 9), the uncooled IR images have most difficulty. However, 
Table 7: RMSE, PSNR and SSIM of cooled emissive IR image - Car engine.

\begin{tabular}{llll}
\hline Enhanced method & RMSE & PSNR & SSIM \\
\hline Nearest & 7.6283 & 30.4822 & 0.9948 \\
Bicubic & 5.8892 & 32.7297 & 0.9954 \\
Bilinear & 6.9002 & 31.3535 & 0.9899 \\
SPIE09 & 8.8736 & 29.1688 & 0.9709 \\
TIP10 & 8.1671 & 29.8894 & 0.9794 \\
TIP13 & 5.6128 & 33.1472 & 0.9845 \\
Proposed Method & 4.8457 & 34.4237 & 0.9981 \\
TIP14 & 4.6653 & 34.7532 & 0.9986 \\
\hline
\end{tabular}

the proposed method still generates high image quality output. They both have best SSIM within eight approaches. Meanwhile, the other seven algorithms have uncertain outcome, but are dependent on desired IR image.

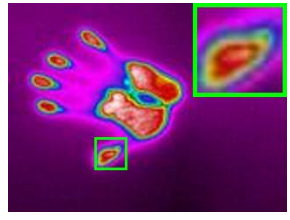

(a)

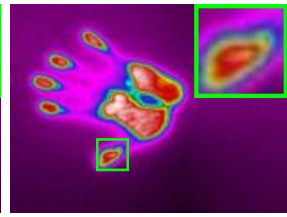

(b)

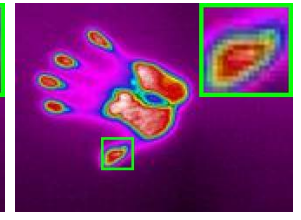

(c)

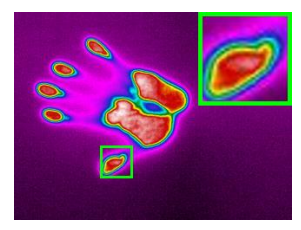

(d)

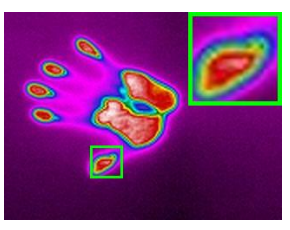

(e)

Figure 8: Hand uncooled emissive IR image. (a) bicubic, (b) bilinear, (c) nearest neighbor, (d) ground image, (e) proposed method

Most of uncooled thermal IR cameras have very low resolution in low-end market. The civil thermal imager features around $60 \times 80$ pixels ( $8 \mathrm{X}$ to $480 \times 640$ ) and enhances the spacial resolution to multiple times digitally. The edge information is lost due to such high enlargement. As shown in Figure 10 (c), the 
Table 8: RMSE, PSNR and SSIM of uncooled emissive IR image - hand.

\begin{tabular}{llll}
\hline Enhanced method & RMSE & PSNR & SSIM \\
\hline Nearest & 7.1789 & 31.0097 & 0.9112 \\
SPIE09 & 7.1489 & 31.0460 & 0.9135 \\
TIP10 & 7.1078 & 31.0961 & 0.9151 \\
Bilinear & 6.7817 & 31.5047 & 0.9174 \\
Bicubic & 6.5315 & 31.8306 & 0.9220 \\
TIP13 & 6.1124 & 32.4066 & 0.9512 \\
TIP14 & 5.1946 & 33.8197 & 0.9624 \\
Proposed Method & 5.1497 & 33.9902 & 0.9688 \\
\hline
\end{tabular}

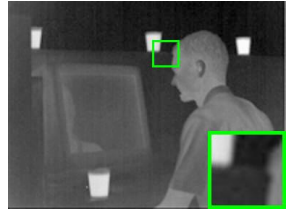

(a)

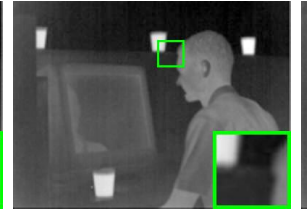

(b)

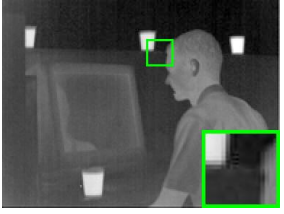

(c)

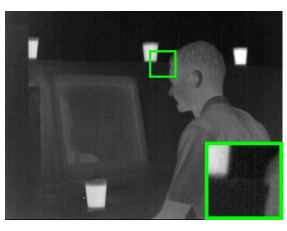

(d)

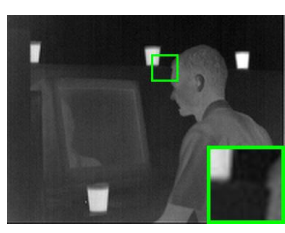

(e)

Figure 9: Human workspace uncooled emissive IR image. (a) bicubic, (b) bilinear, (c) nearest neighbor, (d) ground image, (e) proposed method

output thermal image is blur. However, a high edge model is introduced to strengthen image details in Figure 10 (b). The finger edge can be clearly seen in the reconstruct image. Therefore, the proposed estimator also enhances contrast and sharpness of IR images for high scale factor super resolution. 
Table 9: RMSE, PSNR and SSIM of uncooled emissive IR image - human workspace.

\begin{tabular}{llll}
\hline Enhanced method & RMSE & PSNR & SSIM \\
\hline Nearest & 7.8080 & 30.2800 & 0.8454 \\
Bilinear & 6.7717 & 31.5168 & 0.8471 \\
Bicubic & 6.5582 & 31.7951 & 0.8553 \\
TIP14 & 6.4283 & 31.9689 & 0.8624 \\
SPIE09 & 6.6605 & 31.1541 & 0.9043 \\
TIP10 & 5.3339 & 33.5899 & 0.9093 \\
TIP13 & 6.2652 & 30.7857 & 0.9219 \\
Proposed Method & 6.1802 & 32.7660 & 0.9428 \\
\hline
\end{tabular}

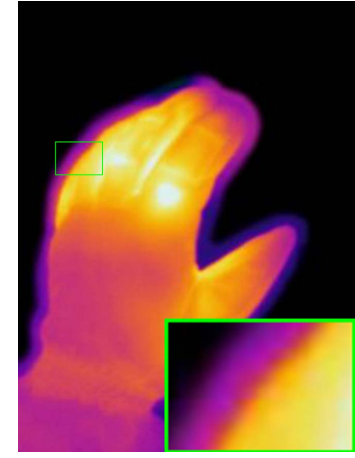

(a)

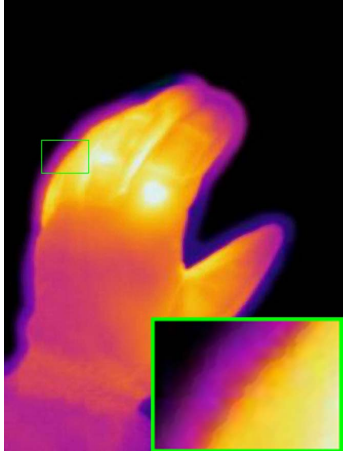

(b)

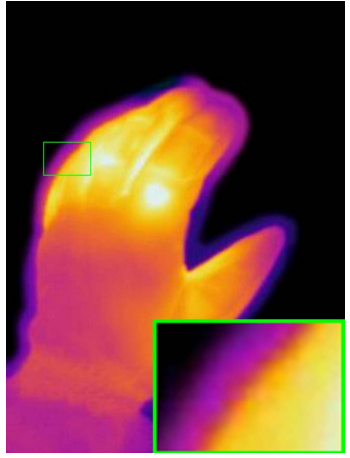

(c)

Figure 10: The high scale factor uncooled thermal image super resolution comparison, (a) bicubic method, (b) proposed method, (c) ground truth image

\section{Conclusion}

The robust super resolution model is proposed for IR image enhancement. In this model, an infrared image is split by smooth part and edge details. The 2D spline based RKHS is used for image base (smooth component) while a modulated heaviside function is applied for image edge. The super resolution is casted into a model based estimation. By computing the coefficients of the redundant basis of low resolution image, it is applied as same in high resolution image for computing. The iterative scheme was proposed to preserve more image 
details. The experimental results showed that the model was robust and easily modified with different IR images. The algorithm runtime is from $10 \mathrm{~s}$ to $50 \mathrm{~s}$ for all reconstructions. Therefore, the computing is still a challenge for this method due to iteration in estimation. However, the parallel computing and hardware accelerating will make the proposed approach fast and easily integrated into consumer electronics. The single image super resolution solves the limitations by sensor array. Besides, the optical diffraction is another obstacle when exploring fine details on target especially on infrared spectrum. Both signal processing and firmware/hardware improvement are required to make progress on physically super resolution.

\section{Acknowledge}

This research work is partially supported under NSF Grants IIS-0713346 and ONR Grants N00014-04-1-0799 and N00014-07-1-0935, the U. S. Army Research

Laboratory, and the U. S. Army Research Office under the grant W911NF-14$1-0327$.

\section{References}

[1] A. Rogalski, K. Chrzanowski, Infrared devices and techniques, OptoElectronics Review 10 (2002) 111-136.

[2] P. W. Kruse, Uncooled Thermal Imaging Arrays, Systems, and Applications, 1st Edition, SPIE Press, Bellingham, WN, 2001.

[3] B. Stuart, Infrared Spectroscopy: Fundamentals and Applications, 1st Edition, Wiley, Hoboken, NJ, 2004.

[4] C. Hildebrandt, C. Raschner, K. Ammer, An overview of recent application of medical infrared thermography in sports medicine in austria, IEEE Engineering in Medicine and Biology 1 (2010) 51-57. 
[5] J. F. Head, F. Wang, C. A. Lipari, R. L. Elliott, The important role of infrared imaging in breast cancer, Inegrative and Comparative Biology 48 (2000) 50-59.

[6] Infrared Imaging Market worth $\$ 8,450$ Million by 2020 (2014).

[1 URL http://www.marketsandmarkets.com/PressReleases/ IR-imaging.asp

[7] A. Rogalski, Infrared detectors: an overview, Infrared Physics \& Technology 43 (2002) 187-210.

[8] J. Piotrowski, A. Rogalski, Uncooled long wavelength infrared photon detectors, Infrared Physics \& Technology 46 (2004) 115-131.

[9] A. Rogalski, J. Antoszewski, L. Faraone, Third generation infrared photodetector arrays, Journal of Applied Physics 105 (2009) 091101.

[10] T. R. Goodall, A. C. Bovik, N. G. Paulter, Tasking on natural statistics of infrared images, IEEE Transactions on Image Processing 25 (2016) 65-79.

[11] M. A. Kinch, Fundamentals of Infrared Detector Materials, 1st Edition, SPIE Press, Bellingham, WN, 2007.

[12] A. Rogalski, Recent progress in infrared detector technologies, Infrared Physics \& Technology 54 (2011) 136-154.

[13] J. G. Harris, Y.-M. Chiang, Nonuniformity correction of infrared image sequences using the constant-statistics constrain, IEEE Transaction on Image Processing 8 (1999) 1148-1151.

[14] S. C. Park, M. K. Park, M. G. Kang, Super-resolution image reconstruction: A technical overview, IEEE Signal Processing Magazine 5 (2003) 21-36.

[15] H. Ur, D. Gross, Improved resolution from sub-pixel shifted pictures, CVGIP: Graphical Models and Image Processing 54 (1992) 181-186. 
[16] S. Kim, W. Su, Recursive high-resolution reconstruction of blurred multiframe images, IEEE Trans. Image Processing 2 (1993) 534-539.

[17] M. Kang, Generalized multichannel image deconvolution approach and its applications, Opt. Eng. 37 (1998) 2953-2964.

[18] R. H. K. Barnard, E. Armstrong, Joint map registration and high-resolution image estimation using a sequence of undersampled images, IEEE Trans. Image Processing 6 (1997) 1621-1633.

[19] H. Chang, D.-Y. Yeung, Y. Xiong, Super-resolution through neighbor embedding, Computer Vision and Pattern Recognition, 2004. Proceedings of the 2004 IEEE Computer Society Conference on 1 (2004) 1-8.

[20] D. Glasner, S. Bagon, M. Irani, Super-resolution from a single image, Computer Vision, 2009 IEEE 12th International Conference on 1 (2009) 349356.

[21] K. Ren, F. Xu, Super-resolution images fusion via compressed sensing and low-rank matrix decomposition, Infrared Physics \& Technology 1 (2015) $61-68$.

[22] M. Sajjad, I. Mehmood, S. W. Baik, Image super-resolution using sparse coding over redundant dictionary based on effective image representations, J. Vis. Commun. Image R. 26 (2015) 50-65.

[23] Y. Zhao, Q. Chen, X. Sui, G. Gu, A novel infrared image super-resolution method based on sparse representation, Infrared Physics \& Technology 71 (2015) 506-513.

[24] A. Panagiotopoulou, V. Anastassopoulos, Super-resolution reconstruction of thermal infrared images, Proceedings of the 4th WSEAS International Conference on REMOTE SENSING 1 (2008) 40-44.

[25] L. Chen, Z. Zhou, N. Xi, R. Yang, B. Song, Z. Sun, C. Su, Super resolution infrared camera using single carbon nanotube photodetector, SENSORS, 2014 IEEE 1 (2014) 1038-1041. 
26 Z. Gang, Z. Kai, S. Wei, Y. Jie, A study on nsct based super-resolution reconstruction for infrared image, TENCON 2013 - 2013 IEEE Region 10 Conference 1 (2013) 1-5.

[27] H. cang Liu, S. tao Li, H. tao Yin, Infrared surveillance image super resolution via group sparse representation, Optics Communications 289 (2013)

[28] S. Dai, Z. Du, H. Xiang, J. Liu, Reconstruction algorithm of superresolution infrared image based on human vision processing mechanism, Front. Optoelectron 8 (2015) 195-202.

[29] W. Gan, C. Ren, X. Wang, X. He, An improving infrared image resolution

[30] P. Ceccato, S. Flasse, S. Tarantola, S. Jacquemound, J.-M. Gregoire, Detecting vegetation leaf water content using reflectance in the optical domain, Remote Sensing of Environment 77 (2001) 22-33.

[31] J. J. Bock, A. E. Lange, Performance of a low-pass filter for far-infrared wavelengths, Applied Optics 34 (1995) 7254C7257.

[32] T. Komatsu, K. Aizawa, T. Igarashi, T. Saito, Signal-processing based method for acquiring very high resolution image with multiple cameras and its theoretical analysis, Proc. Inst. Elec. Eng. 140 (1) (1993) 19-25.

[33] O. Cossairt, M. Gupta, S. K. Nayar, When does computational imaging improve performance, IEEE TRANSACTIONS ON IMAGE PROCESSING 22 (2) (2013) 447-458.

[34] L. jian Deng, W. Guo, T. Huang, Single image super-resolution via an iterative reproducing kernel hilbert space method, IEEE Transactions on Circuits and Systems for Video Technology 1051 (2015) 1-14.

395

[35] G. Wahba, Spline models for observational data, SIAM. Regional Conference Series in Applied Mathematics 59. 
[36] Y. Wang, J. Yang, W. Yin, Y. Zhang, A new alternating minimization algorithm for total variation image reconstruction, SIAM Journal on Imaging Sciences 1 (2008) 248-272.

[37] P. Getreuer, Contour stencils for edge-adaptive image interpolation, Proceedings of SPIE - The International Society for Optical Engineering 7257 (2009) 1-13.

[38] S. Mallat, G. Yu, Super-resolution with sparse mixing estimators, IEEE TRANSACTIONS ON IMAGE PROCESSING 19 (2010) 2889-2900.

[39] W. Dong, L. Zhang, R. Lukac, G. Shi, Sparse representation based image interpolation with nonlocal autoregressive modeling, IEEE Transactions on Image Processing 22 (2013) 1382-1394.

[40] T. Peleg, M. Elad, A statistical prediction model based on sparse representations for single image super-resolution, IEEE Transactions on Image Processing 23 (2014) 2569-2581. 